\title{
Herbal formula YGJDSJ inhibits anchorage- independent growth and induces anoikis in hepatocellular carcinoma Bel-7402 cells
}

\author{
Bing $\mathrm{Hu}^{1,2^{*}}$ (D), Tong Zhang ${ }^{3}$, Hong-Mei An ${ }^{4}$, Jia-Lu Zheng ${ }^{1,2}$, Xia Yan ${ }^{1,2}$ and Xiao-Wei Huang ${ }^{1,2}$
}

\begin{abstract}
Background: Based on clinical medications and related studies, we established a Yang-Gan Jie-Du Sang-Jie (YGJDSJ) herbal formula for hepatocarcinoma treatment. In present study, we evaluated the anti-cancer potential of YGJDSJ on suspension-grown human hepatocellular carcinoma Bel-7402 cells.

Methods: Bel-7402 cells were cultured in poly(2-hydroxyethyl methacrylate) (poly-HEMA) coated plates and treated with YGJDSJ. Anchorage-independent cell growth was detected by cell Counting Kit-8 (CCK-8) assay and soft agar colony formation assay. Anoikis was detected by ethdium homodimer-1 (EthD-1) staining and flow cytometry analysis. Caspases activities were detected by the cleavage of chromogenic substrate. Reactive oxygen species (ROS) was detected by 2',7'-dichlorofluorescin diacetate (DCFH-DA) staining. Protein expression and phosphorylation was identified by western blot. Protein expression was knocked-down by siRNA.

Results: YGJDSJ inhibited the proliferation of Bel-7402 cells in poly-HEMA coated plates and anchorageindependent growth of Bel-7402 cells in soft agar. YGJDSJ also induced anoikis in Bel-7402 cells as indicated by EthD-1 staining and flow cytometry analysis. YGJDSJ activated caspase-3, -8 , and -9 in suspension-grown Bel-7402 cells. The pan-caspase inhibitor Z-VAD-FMK significantly abrogated the effects of YGJDSJ on anoikis in suspension-grown Bel-7402 cells. In addition, YGJDSJ increased ROS in suspension-grown Bel-7402 cells. The ROS scavenger N-acetyl-L-cysteine (NAC) partially attenuated YGJDSJ-induced activation of caspase-3, -8 and -9 and anoikis in suspension-grown Bel-7402 cells. Furthermore, YGJDSJ inhibited expression and phosphorylation of protein tyrosine kinase 2 (PTK2) in suspension-grown Bel-7402 cells. Over-expression of PTK2 significantly abrogated YGJDSJ induced anoikis.
\end{abstract}

Conclusions: YGJDSJ inhibits anchorage-independent growth and induce caspase-mediated anoikis in Bel-7402 cells, and may relate to ROS generation and PTK2 downregulation.

Keywords: Hepatocellular carcinoma, Chinese herb, Anoikis, Caspases, Reactive oxygen species, Protein tyrosine kinase 2

\section{Background}

Hepatocarcinoma is one of the most common malignancies worldwide, and ranks second and sixth as the cause of cancer deaths in men and women, respectively [1]. The treatment options for hepatocarcinoma mostly include surgery, transhepatic artery chemoembolization

\footnotetext{
* Correspondence: beearhu@hotmail.com

'Department of Oncology, Longhua Hospital, Shanghai University of Traditional Chinese Medicine, Shanghai 200032, People's Republic of China ${ }^{2}$ Institute of Traditional Chinese Medicine in Oncology, Shanghai Academy of Traditional Chinese Medicine, Shanghai 200032, People's Republic of China Full list of author information is available at the end of the article
}

(TACE), and targeted therapy. Surgery, comprising either hepatectomy and/or liver transplantation, is the only treatment that can possibly cure hepatocarcinoma. However, only patients with early-stage hepatocarcinoma are eligible for curative surgery. The prognosis for patients with middle and advanced stages of hepatocarcinoma, especially metastatic hepatocarcinoma, remains poor $[2,3]$. Hepatocarcinomas tend to metastasize to the lungs, bones, adrenal glands, and other distant organs via blood circulation. Hepatocarcinomas can also metastasize to the lymph nodes via circulating lymph, including portal 
lymph nodes, periaortic lymph nodes, and supraclavicular lymph nodes $[4,5]$. Cancer metastasis is closely associated with anoikis resistance $[6,7]$.

Anoikis, also known as detachment-induced apoptosis, is the programmed cell death of epithelial cells (including cancer cells) following their detachment from the extracellular matrix (ECM) [8]. Anoikis was first discovered by Frisch and Francis in 1994 [9]. Cancer cells possess a certain degree of resistance to anoikis due to abnormal gene expression or activity that enables them to survive in circulating blood, lymph, or other liquid media, and this ultimately causes metastasis in distant tissues $[6,10]$. However, cancer cells can undergo anoikis upon appropriate drug treatment. For example, fucoxanthinol, 5-azacytidine and foretinib can promote anoikis in cancer cells via different mechanisms [11-13]. Promoting cancer cell anoikis has become an important strategy for preventing cancer metastasis $[14,15]$.

Traditional Chinese medicine (TCM) is an important biomedical resource and plays important role in the treatment of hepatocarcinoma [16]. Based on clinical medications and TCM principles, we have established a herbal formula, Yang-Gan Jie-Du Sang-Jie (YGJDSJ), for hepatocarcinoma treatment. YGJDSJ comprises several Chinese herbs including the fruits of Ligustrum lucidum Ait. (Nü-zhen-zi), Duchesnea indica (Andr.) Focke (She-Mei), Solanum nigrum L. (Long-Kui), Euphorbia helioscopia L. (Ze-Qi), the root of Ranunculus ternatus Thunb. (Mao-Zhua-Cao), the root of Curcuma wenyujin Y. H. Chen et C. Ling (Yü-Jin) and the root of Polygonum cuspidatum Sieb. et Zucc. (Hu-Zhang). Most herbs in YGJDSJ have demonstrated anti-cancer effects in various cancer cells $[16,17]$. In the present study, the effects and possible mechanism of YGJDSJ on anchorage-independent growth and anoikis of hepatocarcinoma cells were evaluated.

\section{Methods}

\section{Chemicals and reagents}

DMEM medium and fetal bovine serum was obtained from Hyclone (Logan, UT). Cell Counting Kit-8 (CCK8) was from Dojindo (Kumamoto, Japan). Caspases activities detection kits, 2',7'-dichlorofluorescin diacetate (DCFH-DA), and N-acetyl-L-cysteine (NAC) were purchased from Beyotime (Haimen, China). Z-VAD-FMK was from R\&D Systems (Minneapolis, MN). Antibodies against protein tyrosine kinase 2 /focal adhesion kinase (PTK2/FAK), p-PTK2 and glyceraldehyde-3-phosphate dehydrogenase (GAPDH) were the product of Cell Signaling Technology (Danvers, MA). Poly(2-hydroxyethyl methacrylate) (poly-HEMA) was produced by SigmaAldrich (St. Louis, MO). CytoSelect ${ }^{\mathrm{TM}}$ 24-Well Anoikis Assay kit was provided by Cell Biolabs (San Diego, CA). Caspase-3, 8 and 9 activity assay kits were provided by Beyotime Institute of Biotechnology (Haimen, China).

\section{Cell culture}

Human hepatocellular carcinoma Bel-7402 cells were obtained from Cell Bank of Type Culture Collection of Chinese Academy of Sciences. Bel-7402 cells were grown in DMEM medium with 10\% FBS and 1\% Pen-Strep, and maintained at a $37{ }^{\circ} \mathrm{C}$ in a humidified incubator with a $5 \% \mathrm{CO}_{2}$ atmosphere. All the cell treatment was did in $10 \%$ FBS condition.

\section{Herb preparation}

The main herbs in YGJDSJ formula (Chinese patent ZL201110145109.0) are the fruits of L. lucidum Ait. (Nü-zhen-zi) $12 \mathrm{~g}$, D. indica (Andr.) Focke (She-Mei) 15 g, S. nigrum L. (Long-Kui) 15 g, Scutellaria barbata D. Don (Ban-Zhi-Lian) 30 g, E. helioscopia L. (Ze-Qi) $15 \mathrm{~g}$, the root of $R$. ternatus Thunb. (Mao-Zhua-Cao) $15 \mathrm{~g}$, the root of $C$. wenyujin Y. H. Chen et C. Ling (YüJin) $15 \mathrm{~g}$ and the root of P. cuspidatum Sieb. et Zucc. (Hu-Zhang) $15 \mathrm{~g}$. The doses of these herbs were based on clinical medication.

All those herbs were from Longhua Hospital according to the original proportion. Herb extraction was performed as described previously [18, 19]. Briefly, herbs were extracted twice with an 8-fold volume of boiling distilled water for $1 \mathrm{~h}$ and the aqueous extracts were collected. The collected aqueous extracts were combined, filtered, centrifuged twice at $12,000 \mathrm{rpm}$ for $30 \mathrm{~min}$ at $4{ }^{\circ} \mathrm{C}$, and the supernatants were collected. The supernatants were then mixed with an equal volume of ethanol and kept at $4{ }^{\circ} \mathrm{C}$ overnight, centrifuged at 12,000 rpm for $30 \mathrm{~min}$ at $4{ }^{\circ} \mathrm{C}$ and the supernatants were collected and lyophilized. Subsequently, the ethanol extracts were dissolved in DMEM medium (400 $\mathrm{mg} / \mathrm{ml})$, sequentially passed through $0.45 \mu \mathrm{m}$ and $0.22 \mu \mathrm{m}$ filters for sterilization, and stored at $-20{ }^{\circ} \mathrm{C}$ until further use.

\section{Anchorage-independent growth assay}

Poly-HEMA, a non-toxic polymer of 2-hydroxyethyl methacrylate, was used for anchorage-independent cell growth in vitro because of its ability to reduce the adhesivity of plastic cell culture plates. Bel-7402 cells in logarithmic growth phase were seeded into poly-HEMA coated 96 -well plate $\left(8 \times 10^{3}\right.$ cells/well). After $24 \mathrm{~h}$ cells were exposed to various doses of YGJDSJ or equal volume of DMEM for $24 \mathrm{~h}$, and cell viability was evaluated by using the CCK- 8 assay according to the manufacturer's instructions. The cell survival rate was calculated as follows: cell survival rate $(\%)=($ experimental $\mathrm{OD}$ value/control OD value) $\times 100 \%$.

For the soft agar colony formation assays, $2 \times 10^{4} \mathrm{log}$ phase Bel-7402 cells were seeded and grown on a plate containing $1 \%$ base agar and $0.6 \%$ top agar, and exposed to different concentrations of YGJDSJ or equal volume of DMEM twice a week for 2 weeks and incubated at $37{ }^{\circ} \mathrm{C}$ 
in a humidified incubator with a $5 \% \mathrm{CO}_{2}$ atmosphere. Colonies were stained with crystal violet a counted under a dissecting microscope. The inhibition of colony formation was calculated as follows: inhibition $(\%)=[($ control colonies - experimental colonies $) /$ control colonies] $\times 100 \%$.

\section{Anoikis assay}

Anoikis was detected by CytoSelect ${ }^{\mathrm{Tw}}$ 24-Well Anoikis Assay kit according to the manufacturer's instructions. Briefly, log-phase Bel-7402 cells $\left(4 \times 10^{4}\right.$ cells/well $)$ were inoculated in poly-HEMA coated 24-well plate. On the second day, the cells were exposed to different dose of YGIDSJ or equal volume of DMEM for $24 \mathrm{~h}$, and stained with ethidium homodimer (EthD-1) at $37{ }^{\circ} \mathrm{C}$ for $1 \mathrm{~h}$. The presence of red EthD-1 fluorescence was monitored under a fluorescence microscope and measured with a fluorescence microplate reader (excitation wavelength $525 \mathrm{~nm}$, emission wavelength $590 \mathrm{~nm}$ ). EthD-1 is a highaffinity fluorescent nucleic acid dye, which can only penetrate membrane damaged dead cells and produces red fluorescence upon binding to nucleic acids, and thus be used to detect cell death in suspension (anoikis).

\section{Flow cytometric analysis}

For apoptosis identification, $2 \times 10^{5} \log$-phase Bel-7402 cells were seeded in poly-HEMA coated 6-well plate. On the second day, cells were treated with different concentrations of YGJDSJ or equal volume of DMEM for $24 \mathrm{~h}$. YGJDSJ treated Bel-7402 cells were collected, stained with Annexin V-FITC and PI as recommended by the manufacturer, and detected in a FACScalibour flow cytometer (Becton Dickinson).

\section{Caspase activity assay}

After treatment with different concentration of YGJDSJ, caspase-3, 8 and 9 activities were measured by the cleavage of the specific chromogenic substrate Ac-DEVDpNA, Ac-IETD-pNA and Ac-LEHD-pNA respectively. The cleavaged yellow pNA are positive correlation with caspase-3, 8 and 9 activities. The presence of yellow pNA was detected with a microplate reader at a wavelength of $405 \mathrm{~nm}$. The results are expressed as the fold change in comparison with the control group. For caspases inhibition, Bel-7402 cells pretreated with Z-VADFMK $(50 \mu \mathrm{mol} / \mathrm{L}, 2 \mathrm{~h})$ were incubated with YGJDSJ for another $24 \mathrm{~h}$.

Measurement of intracellular reactive oxygen species (ROS) Intracellular ROS production was detected by DCFH-DA staining. DCFH-DA is cleaved intracellularly by nonspecific esterases to form DCFH, which is further oxidized by ROS to form the fluorescent compound DCF [20]. Logphase Bel-7402 cells $\left(4 \times 10^{4}\right.$ cells/well $)$ were seeded in
poly-HEMA coated 24-well plate. On the second day, the cells were exposed to different dose of YGJDSJ or equal volume of DMEM for $24 \mathrm{~h}$, and stained with DCFH-DA at $37{ }^{\circ} \mathrm{C}$ for $20 \mathrm{~min}$ in the dark. The presence of DCF fluorescence was observed under a fluorescence microscope and quantitated with a fluorescence microplate reader at excitation wavelength of $488 \mathrm{~nm}$ and emission wavelength of $525 \mathrm{~nm}$. For ROS inhibition, cells were pretreated with NAC ( $50 \mathrm{mmol} / \mathrm{L}$ for $2 \mathrm{~h}$ ), followed by desired YGJDSJ treatment.

\section{Western blot}

Western blots were performed as described previously $[21,22]$. Briefly, collected cells were lysed and subjected to $8-12 \%$ SDS-PAGE, and transferred onto a nitrocellulose membrane (Amersham Biosciences, Buckinghamshire, UK). The transferred membrane were blocked with 5\% non-fat milk, washed, and probed with the indicated antibodies. Blots were then washed and incubated with IRDye 700- or IRDye 800conjugated secondary antibodies (Rockland Immunochemicals, Gilbertsville, PA, USA), and visualized in Odyssey Infrared Imaging System (LI-COR Biosciences, Lincoln, NE, USA).

\section{Plasmid transfection}

For plasmid transfection, Bel-7402 cells were cultured on 6-well plate to $90-95 \%$ confluence, and $4.0 \mu \mathrm{g}$ recombinant human PTK2 eukaryotic expression plasmid or control empty vector (Genechem, Shanghai, China) were introduced into the cells by Lipofectamine ${ }^{\mathrm{rm}} 2000$ according to the manufacturer's recommendations. After $24 \mathrm{~h}$ of transfection, cells were subjected to suspensionculture, YGJDSJ $(200 \mu \mathrm{g} / \mathrm{ml})$ treatment for $24 \mathrm{~h}$, western blot and anoikis assay.

\section{Statistical analyses}

Results are expressed as means \pm standard deviation of at least two independent experiments, each conducted in triplicate. Differences between control and YGJDSJ treatment were analyzed by one-way ANOVA. Differences were considered significant at $P \leq 0.05$.

\section{Results}

YGJDSJ inhibits anchorage-independent growth of Bel-7402 cells

Poly-HEMA-coated culture plates were used to observe the effects of YGJDSJ on anchorage-independent growth in Bel-7402 cells. The results show that YGJDSJ inhibited proliferation of Bel-7402 cells in the poly-HEMA coated plates in a dose-dependent manner $(P<0.01)$ (Fig. 1a).

The effects of YGJDSJ on long-term anchorageindependent Bel-7402 cell growth were further observed 

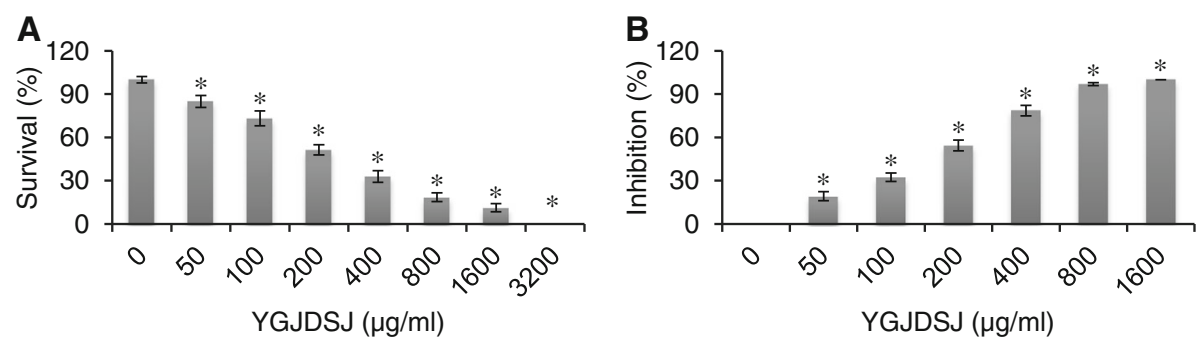

Fig. 1 YGJDSJ inhibited anchorage-independent growth of Bel-7402 cells. a Bel-7402 cells were cultured in poly-HEMA coated 96-well plate and treated with different concentrations of YGJDSJ for 24 h, cell viability was evaluated by CCK-8 assay. b Bel-7402 cells were treated with different dose of YGJDSJ twice a week for 2 weeks in soft-agar colony formation assay. Data shown are representative of three independent experiments. *P $<0.01$, versus control group

using soft agar colony formation assays. The results show that YGJDSJ inhibits colony formation of Bel-7402 cells in a dose-dependent manner $(P<0.01)$ (Fig. 1b). These observations suggest that YGJDSJ can inhibit anchorage-independent growth in Bel-7402 cells.

\section{YGJDSJ induces anoikis in Bel-7402 cells}

Anoikis in Bel-7402 cells was detected with a commercial kit, in which the 24-well culture plate was coated with poly-HEMA, and EthD-1 fluorescence labeling was used for anoikis detection. The results show that after YGJDSJ treatment, Bel-7402 cells absorbed EthD-1 and emitted red fluorescence, which suggesting YGJDSJ induced anoikis in Bel-7402 cells (Fig. 2a and b). Further
Annexin V-FITC/PI double labeling and flow cytometry analysis confirmed that YGJDSJ significantly induced apoptosis in suspension-grown Bel-7402 cell in a dosedependent manner $(P<0.01)$ (Fig. $2 \mathrm{c}$ and $\mathrm{d})$. These results suggest that YGJDSJ induced anoikis in Bel-7402 cells.

\section{YGJDSJ activates caspase activity in Bel-7402 cells}

Similar to that in apoptosis, anoikis is also mediated by the caspase cascade $[6,10,23]$. In this study, the effects of YGJDSJ herbs on caspase activity were detected by commercial kits. As shown in Fig. 3, YGJDSJ significantly activated caspase- $3,-8$, and -9 in suspension-grown Bel-7402 cells in a dose-dependent manner $(P<0.01)$ (Fig. 3a-c). In addition, Z-VAD-FMK, a pan-caspase
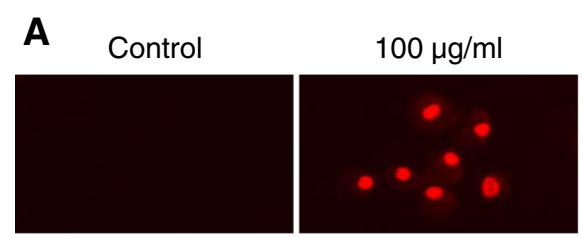

$200 \mu \mathrm{g} / \mathrm{ml}$

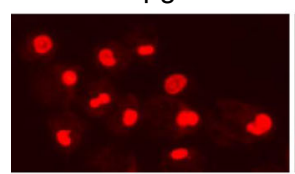

$400 \mu \mathrm{g} / \mathrm{ml}$

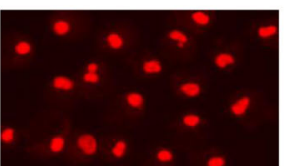

B

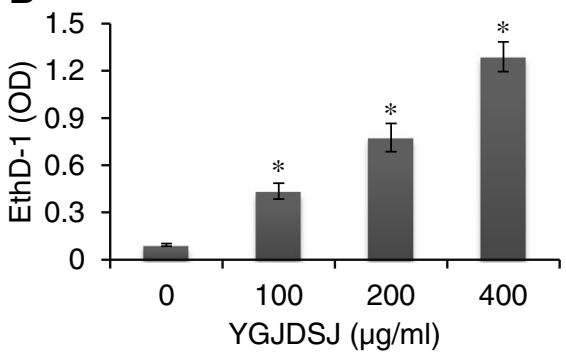

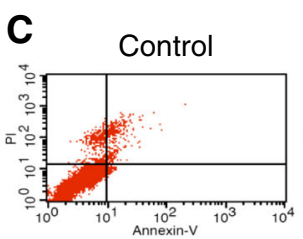

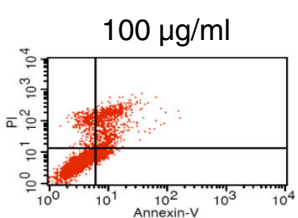

$200 \mu \mathrm{g} / \mathrm{ml}$

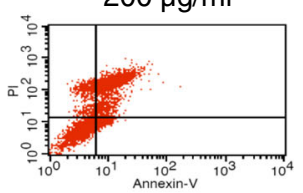

$400 \mu \mathrm{g} / \mathrm{ml}$

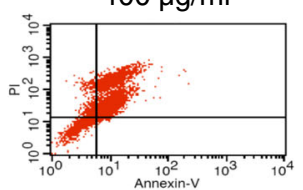

D

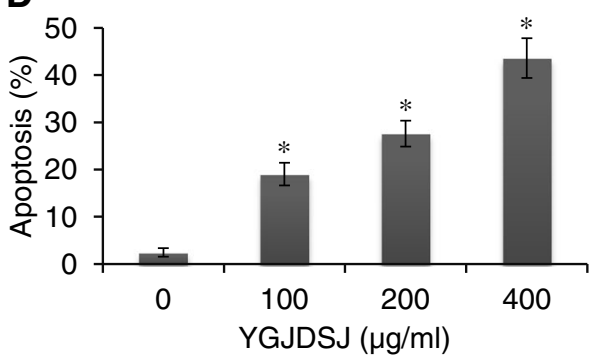

Fig. 2 YGJDSJ induced anoikis in Bel-7402 cells. Suspension-grown Bel-7402 cells were treated with different dose of YGJDSJ for 24 h, stained with EthD-1, and observed under fluorescence microscope $(\times 200)(\mathbf{a})$, and quantitated with a fluorescence microplate reader (b). YGJDSJ treated or untreated Bel-7402 cells were stained with Annexin V-FITC/PI, analyzed in FACScalibour flow cytometer (c), and expressed as mean \pm SD (d). Data illustrated are from three separate experiments. ${ }^{*} P<0.01$, versus control group 

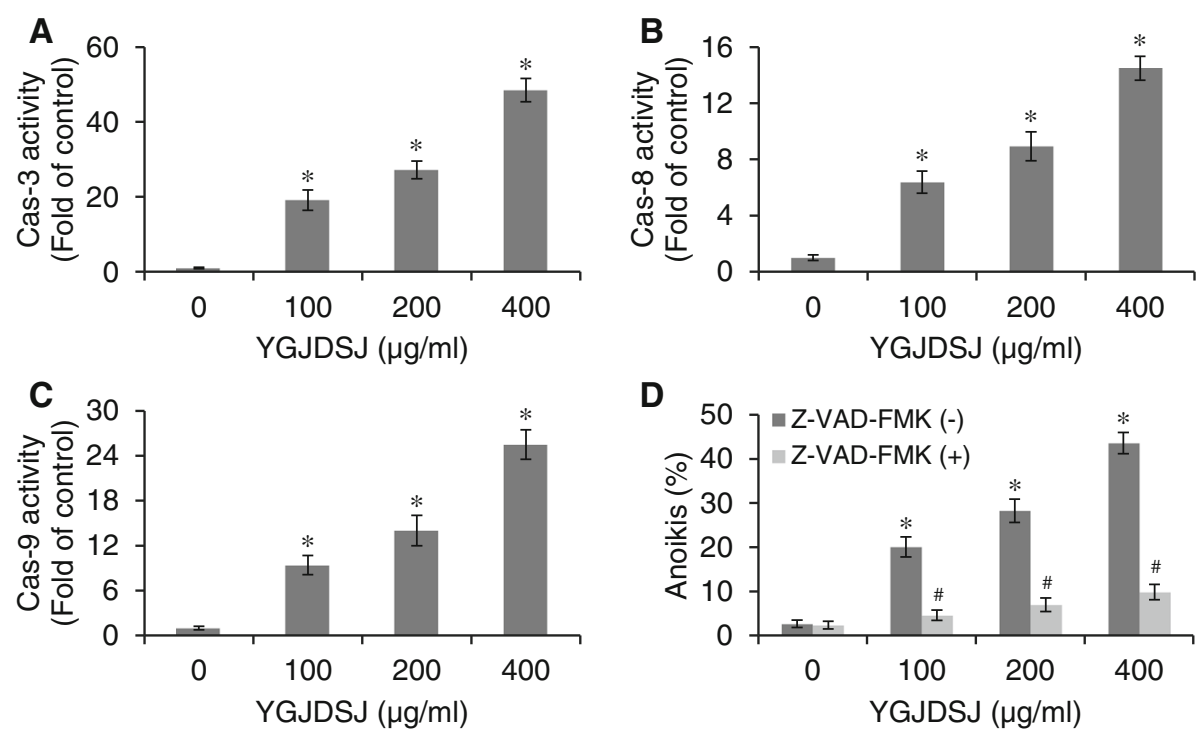

Fig. 3 YGJDSJ activated caspases in Bel-7402 cells. After 24 h YGJDSJ (100-400 $\mu \mathrm{g} / \mathrm{ml}$ ) treatment, caspase-3 (a), caspase-3 (b) and caspase-9 (c) activities in suspension-cultured Bel-7402 cells were detected as described in Materials and Methods. Caspases activities were expressed as fold activation over control. d suspension-cultured Bel-7402 cells were pretreated with Z-VAD-FMK (50 umol/L) for $2 \mathrm{~h}$ before treatment with YGJDSJ for $24 \mathrm{~h}$, stained with Annexin V-FITC/PI and analyzed by flow cytometry. Data presented are from three separate experiments. ${ }^{*} P<0.01$, versus control group; ${ }^{\#} P<0.01$, versus corresponding dose of YGJDSJ treated Z-VAD-FMK (-) group
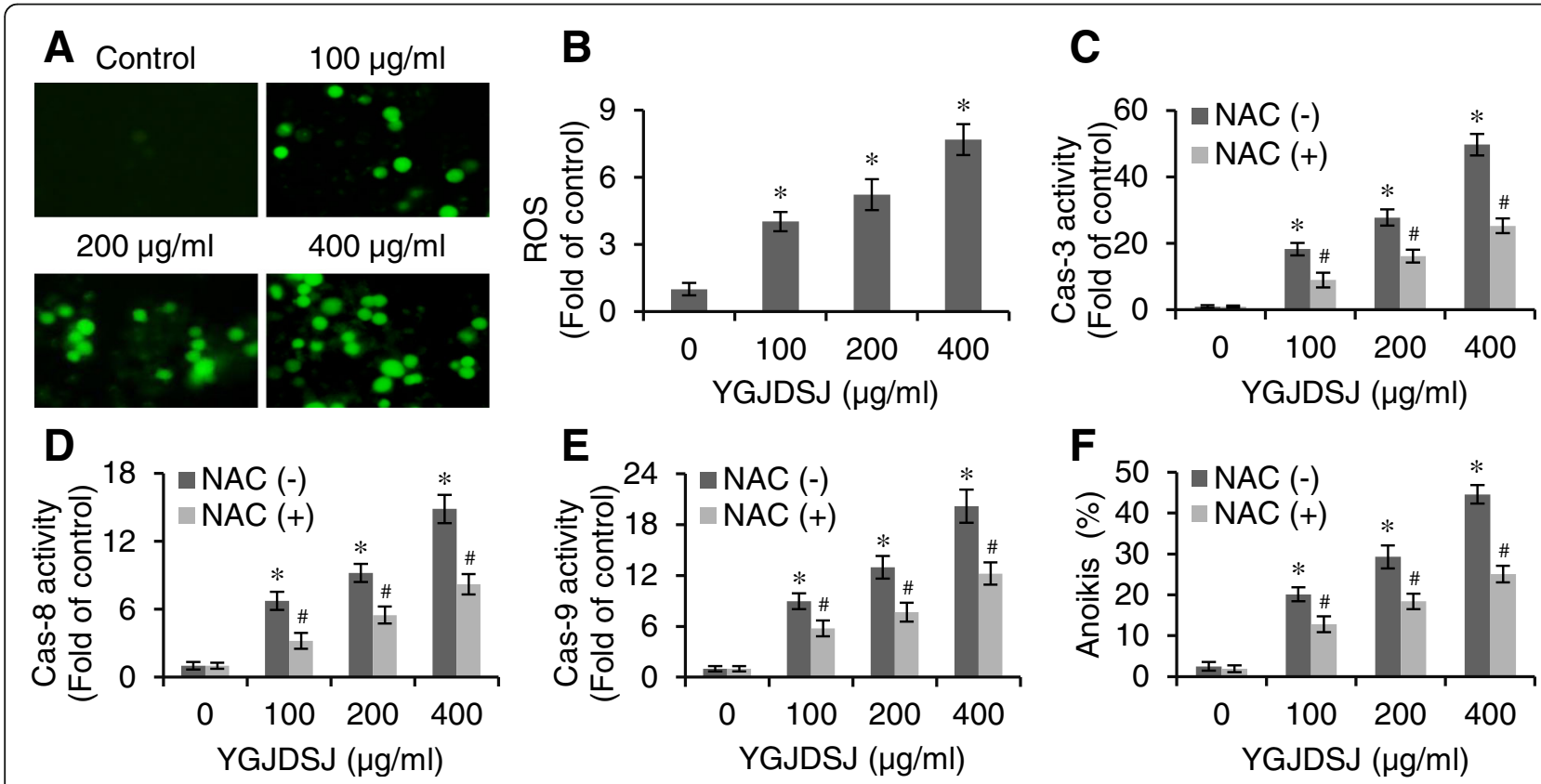

Fig. 4 YGJDSJ increased ROS level in Bel-7402 cells. After 24 h YGJDSJ (100-400 $\mu \mathrm{g} / \mathrm{ml})$ treatment, intracellular ROS production in suspension-cultured Bel-7402 cells was stained with DCFH-DA, observed under fluorescence microscope $(\times 200)(\mathbf{a})$, quantitated with a fluorescence microplate reader and expressed as fold of control (b)Suspension-cultured Bel-7402 cells were pretreated with NAC (50 mmol/L for $2 \mathrm{~h}$ ) for ROS inhibition, followed by YGJDSJ (100-400 jg/ml) treatment for 24 h, and subjected to caspase-3 (c), caspase-8 (d) and caspase-9 (e) activities and anoikis detection (f). Caspases activities were expressed as fold activation over control. Data shown are representative of three independent experiments. ${ }^{*} P<0.01$, versus control group; ${ }^{\#} P<0.01$, versus corresponding dose of YGJDSJ treated NAC (-) group. 

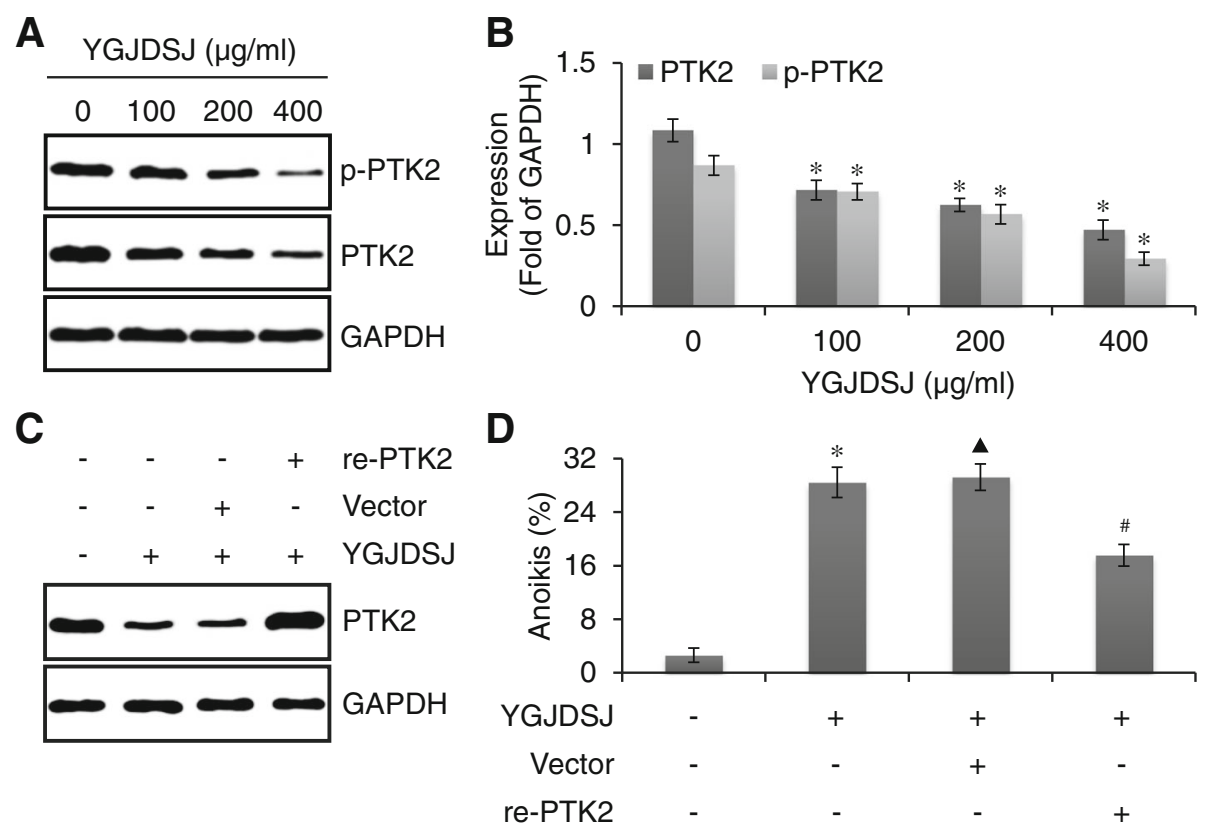

Fig. 5 YGJDSJ inhibited PTK2 expression and phosphorylation in Bel-7402 cells. Suspension-cultured Bel-7402 cells were collected after YGJDSJ treatment, subjected to western blots using indicated antibodies (a), and the expression of proteins were expressed as fold of GAPDH (b). Bel-7402 cells were transfected with recombinant human PTK2 and empty vector, and subjected to suspension-culture, YGJDSJ (200 $\mu \mathrm{g} / \mathrm{ml})$ treatment for $24 \mathrm{~h}$, western blots using indicated antibodies (c) and anoikis detection (d). ${ }^{*} P<0.01$, versus control group; ${ }^{\mathbf{A}} P>0.05$, versus control YGJDSJ group; ${ }^{\#} P<0.01$, versus YGJDSJ treated vector group

inhibitor, significantly abrogated the effect of YGJDSJ on anoikis $(P<0.01)$ (Fig. 3d), which suggests that the effect of YGJDSJ on anoikis in Bel-7402 cells is dependent on caspases.

\section{YGJDSJ induces ROS production in Bel-7402 cells}

It has been reported that ROS is an important mediator of anoikis and its levels increase with caspase activation [24]. In the present study, DCFH-DA staining was used to detect the ROS level. The results show that YGJDSJ significantly induced ROS generation in anchorageindependent grown Bel-7402 cells in a dose-dependent manner $(P<0.01)$ (Fig. 4a and b).

ROS generation was further blocked by NAC to address its role in caspases activation and anoikis. As shown in Fig. 4c-e, blocking ROS production with NAC antagonized the effect of YGJDSJ on caspase-3, -8 , and -9 activation in Bel-7402 cells $(P<0.01)$. NAC also attenuated YGJDSJ induced anoikis in Bel-7402 cells (Fig. 4f) $(P<0.01)$. These observations suggest that ROS contributed to the effects of YGJDSJ on caspases activation and anoikis induction.

\section{YGJDSJ inhibits PTK2 expression}

Tumor cells that detach from the ECM can escape from anoikis via PTK2 activation [25]. In this study, a western blot assay was used to detect the effects of YGJDSJ on the expression and phosphorylation of PTK2 in Bel-7402 cells. As shown in Fig. 5a and b, PTK2 was highly expressed and phosphorylated in anchorage-independent Bel-7402 cells and YGJDSJ inhibited both PTK2 expression and phosphorylation in a dose-dependent manner.

To examine whether down-regulation of PTK2 contribute to YGJDSJ induced anoikis, a recombinant eukaryotic expression plasmid encoding full length of human PTK2 (re-PTK2) was transfected to Bel-7402 cells. The results showed that PTK2 was over-expressed in re-PTK2 transfected Bel-7402 cells (Fig. 5c). PTK2 over-expression partially but significantly abrogated YGJDSJ induced anoikis $(P<0.01)$ (Fig. $5 \mathrm{~d})$. These observations suggested PTK2 down-regulation contributed to YGJDSJ-induced anoikis.

\section{Discussion}

YGJDSJ formula was established in accordance with TCM theories and clinical medications $[16,17,26]$. We observed the basic TCM pathogenesis of liver cancer is liver Yin-deficiency and cancerous toxicity, and proposed a new therapeutic principle for liver cancer treatment, which is nourishing the liver-Yin (Yang-Gan, YG), detoxifying (Jie-Du, JD) and resolving tumor masses (Sang-Jie, SJ). Chinese herbs commonly medicated in our clinical practice with YG, JD and SJ efficacy were selected and combined as an YGJDSJ formula.

Most herbs in YGJDSJ have been confirmed to have anti-cancer effects. L. lucidum Ait. fruit (Nü-zhen-zi) is 
one of the most frequently used herbs in liver cancer treatment. L. lucidum Ait. fruit (Nü-zhen-zi) can inhibit proliferation, activate caspase- $3,-8$ and -9 to induce apoptosis and inhibit RB phosphorylation to promote cell senescence in hepatocellular carcinoma cells [19]. L. lucidum Ait. fruit (Nü-zhen-zi) in YGJDSJ was use for nourishing the liver-Yin.

S. nigrum L. (Long-Kui) can induce apoptosis and autophagy, and arrest the cell cycle in G2/M phase in hepatocellular carcinoma cells $[27,28]$. In ovarian cancer, $D$. indica (Andr.) Focke (She-Mei) can promote cell apoptosis, arrest the cell cycle in the $\mathrm{S}$ phase, and inhibit tumor growth [29]. S. barbata D. Don (Ban-Zhi-Lian) can induce apoptosis via the mitochondrial pathway in liver cancer cells [30]. S. nigrum L. (Long-Kui), D. indica (Andr.) Focke (She-Mei) and S. barbata D. Don (Ban-Zhi-Lian) in YGJDSJ were used for detoxifying.

E. helioscopia L. (Ze-Qi) can promote apoptosis as well as inhibit growth, invasion, and metastasis of hepatocellular carcinoma [31, 32]. C. wenyujin Y. H. Chen et C. Ling (YüJin) extracts can inhibit proliferation of colorectal carcinoma cells [33]. P. cuspidatum Sieb. et Zucc. (Hu-Zhang) shows anti-cancer effects against oral carcinoma, hepatocellular carcinoma, melanoma, and other tumor cells $[18,34,35]$. E. helioscopia L. (Ze-Qi), R. ternatus Thunb. (Mao-Zhua-Cao), C. wenyujin Y.H.Chen et C.Ling (Yü-Jin) and P. cuspidatum Sieb. et Zucc. (Hu-Zhang) in YGJDSJ were applied for resolving tumor masses. Thus, YGJDSJ is a modern herbal formula with anti-cancer effect in line with TCM theory. Herbs in YGJDSJ coordinate with each other in the aspect of traditional TCM efficacy. However, the additive or synergistic pharmacological effects of these herbs need further investigation.

P. cuspidatum Sieb. et Zucc. (Hu-Zhang) and D. indica (Andr.) Focke (She-Mei) have demonstrated anoikisinducing effects in cancer cells $[18,36]$. Anokis is usually investigated in cell models. Poly-HEMA coating and soft agar colony formation assays are classical anchorageindependent growth models, in which cell grow in a suspension and/or anchorage-independent manner $[9,18,37$, 38]. In the present study, it was shown that YGJDSJ inhibited the growth of Bel-7402 cells in poly-HEMA-coated plates and soft agar, which suggests that YGJDSJ can inhibit the anchorage-independent growth of Bel-7402 cells. Further EthD-1 staining and flow cytometric analysis showed that YGJDSJ could induce anoikis in Bel-7402 cells.

Anoikis is a special type of apoptosis, which is also mediated by the caspase cascade $[6,10,23,39]$. Cells detached from the ECM can activate caspase- 9 or -8 via intrinsic or extrinsic pathways, thereby activating the apoptosisexecuting protease caspase- 3 and inducing anoikis. Results of this study revealed that YGJDSJ activated caspase- $3,-8$, and -9 in suspension-grown Bel-7402 cells. Z-VAD-FMK, a pan-caspase inhibitor, could abrogate the effect of
YGJDSJ on anoikis in Bel-7402 cells. These findings suggest that YGJDSJ can induce Bel-7402 cell anoikis in a caspases-dependent manner via intrinsic and extrinsic apoptotic pathways.

ROS, including oxygen ions, peroxides, and oxygencontaining free radicals, is produced by cells during aerobic metabolism. High level of ROS can trigger apoptosis via intrinsic and/or extrinsic pathways [40, 41]. Natural products, such as P. cuspidatum Sieb. et Zucc. (Hu-Zhang), emodin, curcumin, and grape seed extracts, can induce anoikis in cancer cells by increasing intracellular ROS levels [11, 18, $42,43]$. In the present study, it was found that YGJDSJ induced ROS generation in Bel-7402 cells. In addition, ROS scavenger NAC could antagonize the effects of YGJDSJ on caspase-3, -8 , and- 9, and on anoikis in Bel-7402 cells. These findings suggest that ROS generation contributed to YGJDSJ activated caspases and induced anoikis.

Epithelial cells, including epithelial cancer cells, adhere to the ECM, form focal adhesion plaques mediated by integrin, and thereby promote PTK2 conformational changes and activation; this results in the production of survival signals for cell growth and proliferation through downstream signal transduction [23, 44, 45]. Detachment from ECM will result in anoikis. Cancer cells can escape anoikis by modulating PTK2 activity [25]. On the other hand, down-regulation of PTK2 expression can promote cancer cell anoikis [46, 47]. Results from this study demonstrate that YGJDSJ herbs could inhibit expression and phosphorylation of PTK2, and PTK2 overexpression could attenuate the effect of YGJDSJ on anoikis in Bel7402 cells, suggesting that down-regulation of PTK2 contributes to YGJDSJ-induced anoikis.

It has been reported $\mathrm{H}_{2} \mathrm{O}_{2}$ or AKT inhibitor SC66 induced ROS can inhibit FAK (PTK2) phosphorylation $[48,49]$. On the other hand, FAK inhibitor MSN-HCD may upregulate ROS level in glioma cells [50]. In addition, depression of FAK by shRNA induces apoptosis in rat osteosarcoma cells through activation of caspase-3, 7 and 9 [51]. Loss of Rb-E2F by displacement of promoters induces apoptosis through inactivation of FAK and activation of caspase-8 [52]. Doxazosin induces anoikis in prostate cancer cells via activation of caspase3 and a reduction of FAK [53]. These observations suggest that YGJDSJ induced ROS and inhibited FAK may contribute to each other and both contribute to caspases activation.

\section{Conclusion}

In summary, the present study showed that YGJDSJ inhibited suspension growth of Bel-7402 cells. YGJDSJ increased ROS generation, activated caspase- $3,-8$, and -9 , and down-regulated PTK2 and thus induced anoikis in Bel-7402 cells. Since anoikis is related to cancer metastasis and cell survival in blood or lymphatic circulation, 
the effects of YGJDSJ against hepatocarcinoma metastasis and hepatocarcinoma cells in blood or lymphatic circulation, such as circulating tumor cells, are worthy of further study.

\section{Abbreviations}

CCK-8: Cell counting kit-8; DCFH-DA: 2',7'-dichlorofluorescin diacetate; DMEM: Dulbecco's modified Eagle's medium; ECM: Extracellular matrix; EthD1: Ethidium homodimer-1; FAK: Focal adhesion kinase; FBS: Fetal bovine serum; GAPDH: Glyceraldehyde-3-phosphate dehydrogenase; NAC: N-acetylL-cysteine; poly-HEMA: poly(2-hydroxyethyl methacrylate); PTK2: Protein tyrosine kinase 2; ROS: Reactive oxygen species; TACE: Transhepatic artery chemoembolization; TCM: Traditional Chinese medicine; YGJDSJ: Yang-Gan Jie-Du San-Jie herbal formula

\section{Acknowledgements}

Not applicable.

\section{Funding}

This study was supported by Program from Science \& Technology Commission of Shanghai Municipality (16401902500), and Three-year Action Program of Shanghai Municipality for Traditional Chinese Medicine (ZY3-CCCX-3-3025).

\section{Availability of data and materials}

All data and materials are contained and described in the main paper.

\section{Authors' contributions}

BH conceived and designed the study. BH, TZ, HMA, JLZ, XY and XWH carried out the experiment. HMA analyzed the data. BH drafted and revised the manuscript. All authors read and approved the final manuscript.

\section{Ethics approval and consent to participate}

Not applicable.

\section{Consent for publication}

Not applicable.

\section{Competing interests}

The authors declare that they have no competing interests.

\section{Publisher's Note}

Springer Nature remains neutral with regard to jurisdictional claims in published maps and institutional affiliations.

\section{Author details}

'Department of Oncology, Longhua Hospital, Shanghai University of Traditional Chinese Medicine, Shanghai 200032, People's Republic of China. ${ }^{2}$ Institute of Traditional Chinese Medicine in Oncology, Shanghai Academy of Traditional Chinese Medicine, Shanghai 200032, People's Republic of China. ${ }^{3}$ Experiment Center for Teaching and Learning, Shanghai University of Traditional Chinese Medicine, Shanghai 201204, People's Republic of China. ${ }^{4}$ Department of Science \& Technology, Longhua Hospital, Shanghai University of Traditional Chinese Medicine, Shanghai 200032, People's Republic of China.

Received: 11 July 2017 Accepted: 8 January 2018

Published online: 16 January 2018

\section{References}

1. Torre LA, Bray F, Siegel RL, Ferlay J, Lortet-Tieulent J, Jemal A. Global cancer statistics, 2012. CA Cancer J Clin. 2015:65(2):87-108.

2. Grandhi MS, Kim AK, Ronnekleiv-Kelly SM, Kamel IR, Ghasebeh MA, Pawlik TM. Hepatocellular carcinoma: from diagnosis to treatment. Surg Oncol. 2016;25(2):74-85.

3. Zhuang L, Wen T, Xu M, Yang J, Wang W, Wu H, et al. Sorafenib combined with hepatectomy in patients with intermediate-stage and advanced hepatocellular carcinoma. Arch Med Sci. 2017;13(6):1383-93.

4. Duseja A. Staging of hepatocellular carcinoma. J Clin Exp Hepatol. 2014; 4(Suppl 3):S74-9.
5. Yüksel Ş, Boylu Akyerli C, Cengiz Yakıııer M. Angiogenesis, invasion, and metastasis characteristics of hepatocellular carcinoma. J Gastrointest Cancer. 2017; https://doi.org/10.1007/s12029-017-9962-5.

6. Simpson CD, Anyiwe K, Schimmer AD. Anoikis resistance and tumor metastasis. Cancer Lett. 2008;272(2):177-85.

7. Sun B, Hu C, Yang Z, Zhang X, Zhao L, Xiong J, et al. Midkine promotes hepatocellular carcinoma metastasis by elevating anoikis resistance of circulating tumor cells. Oncotarget. 2017;8(20):32523-35.

8. Grossmann J. Molecular mechanisms of "detachment-induced apoptosisAnoikis". Apoptosis. 2002;7(3):247-60.

9. Frisch SM, Francis $\mathrm{H}$. Disruption of epithelial cell-matrix interactions induces apoptosis. J Cell Biol. 1994;124(4):619-26.

10. Paoli P, Giannoni E, Chiarugi P. Anoikis molecular pathways and its role in cancer progression. Biochim Biophys Acta. 2013;1833(12):3481-98.

11. Terasaki M, Maeda H, Miyashita K, Mutoh M. Induction of anoikis in human colorectal cancer cells by fucoxanthinol. Nutr Cancer. 2017;69(7):1043-52.

12. Chang HW, Wang HC, Chen CY, Hung TW, Hou MF, Yuan SS, et al. 5azacytidine induces anoikis, inhibits mammosphere formation and reduces metalloproteinase 9 activity in MCF-7 human breast cancer cells. Molecules. 2014;19(3):3149-59.

13. Zillhardt M, Park SM, Romero IL, Sawada K, Montag A, Krausz T, et al. Foretinib (GSK1363089), an orally available multikinase inhibitor of c-met and VEGFR-2, blocks proliferation, induces anoikis, and impairs ovarian cancer metastasis. Clin Cancer Res. 2011;17(12):4042-51.

14. Sakamoto S, Kyprianou N. Targeting anoikis resistance in prostate cancer metastasis. Mol Asp Med. 2010;31(2):205-14.

15. Song KJ, Jeon SK, Moon SB, Park JS, Kim JS, Kim J, et al. Lectin from Sambucus sieboldiana abrogates the anoikis resistance of colon cancer cells conferred by $\mathrm{N}$-acetylglucosaminyltransferase $\mathrm{V}$ during hematogenous metastasis. Oncotarget. 2017;8(26):42238-51.

16. Hu B, Wang SS, Du Q. Traditional Chinese medicine for prevention and treatment of hepatocarcinoma: from bench to bedside. World I Hepatol. 2015;7(9):1209-32

17. Hu B, An HM, Wang SS, Chen JJ, Xu L. Preventive and therapeutic effects of Chinese herbal compounds against hepatocellular carcinoma. Molecules. 2016;21(2):142.

18. Hu B, An HM, Shen KP, Song HY, Deng S. Polygonum cuspidatum extract induces anoikis in hepatocarcinoma cells associated with generation of reactive oxygen species and downregulation of focal adhesion kinase. Evid Based Complement Alternat Med. 2012;2012:607675.

19. Hu B, Du Q, Deng S, An HM, Pan CF, Shen KP, et al. Ligustrum lucidum Ait. Fruit extract induces apoptosis and cell senescence in human hepatocellular carcinoma cells through upregulation of p21. Oncol Rep. 2014;32(3):1037-42.

20. Smith JA, Weidemann MJ. Further characterization of the neutrophil oxidative burst by flow cytometry. J Immunol Methods. 1993;162(2):261-8.

21. Hu B, Shen KP, An HM, Wu Y, Du Q. Aqueous extract of Curcuma aromatica induces apoptosis and G2/M arrest in human colon carcinoma LS-174-T cells independent of p53. Cancer Biother Radiopharm. 2011;26(1):97-104.

22. $H u B$, An HM, Shen KP, Xu L, Du Q, Deng S, et al. Modified Yi guan Jian, a Chinese herbal formula, induces anoikis in Bel-7402 human hepatocarcinoma cells in vitro. Oncol Rep. 2011:26(6):1465-70.

23. Chiarugi P, Giannoni E. Anoikis: a necessary death program for anchoragedependent cells. Biochem Pharmacol. 2008:76(11):1352-64.

24. Li AE, Ito H, Rovira II, Kim KS, Takeda K, Yu ZY, et al. A role for reactive oxygen species in endothelial cell anoikis. Circ Res. 1999:85(4):304-10.

25. Liu G, Meng X, Jin Y, Bai J, Zhao Y, Cui X, et al. Inhibitory role of focal adhesion kinase on anoikis in the lung cancer cell A549. Cell Biol Int. 2008; 32(6):663-70

26. Du Q, Hu B, Shen KP. Pathogenesis and treatment of hepatocellular carcinoma in traditional Chinese medicine. World I Integr Trad West Med. 2010;5(9):814-7. Chinese

27. Lin HM, Tseng HC, Wang CJ, Chyau CC, Liao KK, Peng PL, et al. Induction of autophagy and apoptosis by the extract of Solanum nigrum Linn in HepG2 cells. J Agric Food Chem. 2007;55(9):3620-8.

28. Wang HC, Chung PJ, Wu CH, Lan KP, Yang MY, Wang CJ. Solanum nigrum L. polyphenolic extract inhibits hepatocarcinoma cell growth by inducing G2/ M phase arrest and apoptosis. J Sci Food Agric. 2011;91(1):178-85.

29. Peng B, Hu Q, Liu X, Wang L, Chang Q, Li J, et al. Duchesnea phenolic fraction inhibits in vitro and in vivo growth of cervical cancer through induction of apoptosis and cell cycle arrest. Exp Biol Med (Maywood). 2009;234(1):74-83. 
30. Gao J, Lu WF, Dai ZJ, Lin S, Zhao Y, Li S, et al. Induction of apoptosis by total flavonoids from Scutellaria barbata D. Don in human hepatocarcinoma MHCC97-H cells via the mitochondrial pathway. Tumour Biol. 2014;35(3): 2549-59.

31. Cheng J, Han W, Wang Z, Shao Y, Wang Y, Zhang Y, et al. Hepatocellular carcinoma growth is inhibited by Euphorbia helioscopia L. extract in nude mice xenografts. Biomed Res Int. 2015;2015:601015.

32. Wang ZY, Liu HP, Zhang YC, Guo LQ, Li ZX, et al. Anticancer potential of Euphorbia helioscopia L extracts against human cancer cells. Anat Rec (Hoboken). 2012;295(2):223-33.

33. Wang $X$, Jiang $Y$, Hu D. Optimization and in vitro antiproliferation of Curcuma wenyujin's active extracts by ultrasonication and response surface methodology. Chem Cent J. 2016;10:32.

34. Shin JA, Shim JH, Jeon JG, Choi KH, Choi ES, Cho NP, et al. Apoptotic effect of Polygonum Cuspidatum in oral cancer cells through the regulation of specificity protein 1. Oral Dis. 2011;17(2):162-70.

35. Lee CC, Chen YT, Chiu CC, Liao WT, Liu YC, David Wang HM. Polygonum cuspidatum extracts as bioactive antioxidaion, anti-tyrosinase, immune stimulation and anticancer agents. J Biosci Bioeng. 2015;119(4):464-9.

36. Hu B, Shen KP, Shi XF, Deng S, Wei MM. An experimental study on effects of Duchesnea indica on anoikis in human colonic carcinoma RKO cells. World J Integr Trad West Med. 2013;8(1):69-72. Chinese.

37. Bretland AJ, Lawry J, Sharrard RM. A study of death by anoikis in cultured epithelial cells. Cell Prolif. 2001;34(4):199-210.

38. Sakamoto S, McCann RO, Dhir R, Kyprianou N. Talin1 promotes tumor invasion and metastasis via focal adhesion signaling and anoikis resistance. Cancer Res. 2010;70(5):1885-95.

39. Coates JM, Galante JM, Bold RJ. Cancer therapy beyond apoptosis: autophagy and anoikis as mechanisms of cell death. I Surg Res. 2010; 164(2):301-8

40. Circu ML, Aw TY. Reactive oxygen species, cellular redox systems, and apoptosis. Free Radic Biol Med. 2010;48(6):749-62.

41. Lau AT, Wang Y, Chiu JF. Reactive oxygen species: current knowledge and applications in cancer research and therapeutic. J Cell Biochem. 2008;104(2): 657-67

42. Cai J, Niu X, Chen Y, Hu Q, Shi G, Wu H, et al. Emodin-induced generation of reactive oxygen species inhibits RhoA activation to sensitize gastric carcinoma cells to anoikis. Neoplasia. 2008;10(1):41-51.

43. Kaur M, Agarwal R, Agarwal C. Grape seed extract induces anoikis and caspase-mediated apoptosis in human prostate carcinoma LNCaP cells: possible role of ataxia telangiectasia mutated-p53 activation. Mol Cancer Ther. 2006;5(5):1265-74.

44. Nagaprashantha LD, Vatsyayan R, Lelsani PC, Awasthi S, Singhal SS. The sensors and regulators of cell-matrix surveillance in anoikis resistance of tumors. Int J Cancer. 2011;128(4):743-52.

45. Zouq NK, Keeble JA, Lindsay J, Valentijn AJ, Zhang L, Mills D, et al. FAK engages multiple pathways to maintain survival of fibroblasts and epithelia: differential roles for paxillin and p130Cas. J Cell Sci. 2009;122(Pt 3):357-67.

46. Duxbury MS, Ito H, Zinner MJ, Ashley SW, Whang EE. Focal adhesion kinase gene silencing promotes anoikis and suppresses metastasis of human pancreatic adenocarcinoma cells. Surgery. 2004;135(5):555-62.

47. Casanova I, Parreño M, Farré L, Guerrero S, Céspedes MV, Pavon MA, et al. Celecoxib induces anoikis in human colon carcinoma cells associated with the deregulation of focal adhesions and nuclear translocation of p130Cas. Int J Cancer. 2006;118(10):2381-9.

48. Song H, Cha MJ, Song BW, Kim IK, Chang W, Lim S, et al. Reactive oxygen species inhibit adhesion of mesenchymal stem cells implanted into ischemic myocardium via interference of focal adhesion complex. Stem Cells. 2010;28(3):555-63.

49. Cusimano A, Puleio R, D'Alessandro N, Loria GR, McCubrey JA, Montalto G, et al. Cytotoxic activity of the novel small molecule AKT inhibitor SC66 in hepatocellular carcinoma cells. Oncotarget. 2015;6(3):1707-22.

50. Thiyagarajan V, Lin SX, Lee CH, Weng CF. A focal adhesion kinase inhibitor 16-hydroxy-cleroda-3,13-dien-16,15-olide incorporated into enteric-coated nanoparticles for controlled anti-glioma drug delivery. Colloids Surf B Biointerfaces. 2016;141:120-31.

51. Yang S, Wang L, Kong Q. Depression of focal adhesion kinase induces apoptosis in rat osteosarcoma OSR-6 cells in a caspase-dependent pathway. Cell Biochem Biophys. 2014;70(2):765-70.
52. Lieman JH, Worley LA, Harbour JW. Loss of Rb-E2F repression results in caspase-8-mediated apoptosis through inactivation of focal adhesion kinase. J Biol Chem. 2005;280(11):10484-90.

53. Walden PD, Globina Y, Nieder A. Induction of anoikis by doxazosin in prostate cancer cells is associated with activation of caspase-3 and a reduction of focal adhesion kinase. Urol Res. 2004;32(4):261-5.

\section{Submit your next manuscript to BioMed Central and we will help you at every step:}

- We accept pre-submission inquiries

- Our selector tool helps you to find the most relevant journal

- We provide round the clock customer support

- Convenient online submission

- Thorough peer review

- Inclusion in PubMed and all major indexing services

- Maximum visibility for your research

Submit your manuscript at www.biomedcentral.com/submit
Biomed Central 